\title{
On the existence of generalized weak solutions to discontinuous fuzzy differential equations
}

\author{
Ya-Bin Shao ${ }^{a}$, Zeng-Tai Gong ${ }^{\text {,**, }}$ Zi-Zhong Chen $^{c}$ \\ a School of Science, Chongqing University of Posts and Telecommunications, 400065 Nanan, Chongqing, People's Republic of China. \\ ${ }^{b}$ College of Mathematics and Statistics, Northwest Normal University, 730070 Lanzhou, Gansu, People's Republic of China. \\ ${ }^{c}$ College of Computer Science and Technology, Chongqing University of Posts and Telecommunications, 400065 Nanan, Chongqing, \\ People's Republic of China.
}

Communicated by X. Z. Liu

\begin{abstract}
In this paper, by means of replacing the Lebesgue integrability of support functions with its Henstock integrability, the definitions of the Henstock-Pettis integral of n-dimensional fuzzy-number-valued functions are defined. In addition, the controlled convergence theorems for such integrals are considered. As the applications of these integrals, we provide some existence theorems of generalized weak solutions to initial value problems for the discontinuous fuzzy differential equations under the strong GH-differentiability. (C)2017 All rights reserved.
\end{abstract}

Keywords: Fuzzy number, fuzzy Henstock-Pettis integral, convergence theorem, discontinuous fuzzy differential equation, generalized weak solution.

2010 MSC: $03 E 72,26 \mathrm{~A} 39,46 \mathrm{G} 05,34 \mathrm{~A} 07$.

\section{Introduction}

It is well-known that the Henstock integral includes the Riemann, improper Riemann, Lebesgue and Newton integrals [21,25]. The Henstock integral is more powerful and simpler than the Lebesgue integral. It is also equal to the Denjoy and Perron integrals [26]. In the theory of integrals, there are some integrals based on the Banach space-valued functions such as Pettis and Bochner integrals [13, 14, 26, 29]. The integrals of fuzzy-number-valued functions, as a natural generalization of set-valued functions, have been discussed by Puri and Ralescu [27], Kaleva [22], and other authors [36, 37, 40]. Recently, Wu and Gong [15, $18,19]$ have combined the fuzzy set theory and nonabsolute integration theory, and discussed the fuzzy Henstock integrals of fuzzy-number-valued functions which extended Kaleva [22] integration. In order to complete the theory of fuzzy calculus and to meet the solving need of transferring a fuzzy differential equation into a fuzzy integral equation, we $[15,17]$ has defined the strong fuzzy Henstock integrals and

\footnotetext{
${ }^{*}$ Corresponding author

Email addresses: yb-shao@163.com (Ya-Bin Shao), zt-gong@163.com (Zeng-Tai Gong), chenzz@cqupt.edu.cn (Zi-Zhong Chen)

doi:10.22436/jnsa.010.12.12
} 
discussed some of their properties and the controlled convergence theorem. However, for a fuzzy-valued function in the $n$-dimensional fuzzy number space $E^{n}$, the integral and its characteristic theorems have not been defined or discussed. In this paper, by means of replacing the Lebesgue integrability of support functions with its Henstock integrability, we discuss the Henstock-Pettis integral of $n$-dimensional fuzzynumber-valued functions and its controlled convergence theorems.

Differential equations are used for modeling various physics. Unfortunately, many problems are too dynamical and complicated and an accurate differential equation model for such problems requires complex and time consuming algorithms hardly implementable in practice. Thus, a usage of fuzzy mathematics seems to be appropriate. The Cauchy problems for fuzzy differential equations have been studied by several authors $[2,4-8,16,22,27,30]$ on the metric space $\left(E^{n}, D\right)$ of normal fuzzy convex set with the distance $\mathrm{D}$ given by the maximum of the Hausdorff distance between the corresponding level sets. In 2002, Xue and $\mathrm{Fu}$ [39] established solutions to fuzzy differential equations with right-hand side functions satisfying Caratheodory conditions on a class of Lipschitz fuzzy sets. However, there are discontinuous systems in which the right-hand side functions $f:[a, b] \times E^{n} \rightarrow E^{n}$ are not integrable in the sense of Kaleva [22] on certain intervals and their solutions are not absolute continuous functions. So, in [31-35], we used the strong fuzzy Henstock integral [17], and dealt with the Cauchy problem of discontinuous fuzzy systems. In this paper, according to the idea of [9] and using the concept of generalized differentiability [3], we will deal with the Cauchy problem of discontinuous fuzzy systems and the right side-hand functions are fuzzy Henstock-Pettis integrable.

The rest of the paper is organized as follows. In Section 2, we give some basic concepts and preliminary results and the definition of the Henstock-Pettis integral for fuzzy-number-valued functions. In Section 3, we prove a controlled convergence theorem for the fuzzy Henstock-Pettis integrals. As the applications of these integrals, we deal with the Cauchy problem of discontinuous fuzzy systems. And in Section 4, we present some concluding remarks.

\section{Preliminaries}

Let $T$ be the closed interval on the real line R, i.e., $T=[a, b](a, b \in R) .|T|$ denotes the length of $T$. If there exist $T_{i} \subseteq T, \xi_{i} \in T_{i}(i=1,2, \cdots, k)$, such that $\bigcup_{i=1}^{k} T_{i}=T$ (where $T_{1}, T_{2}, \cdots, T_{k}$ are nonoverlapping subintervals of $T)$, then a collection $\left\{\left(\xi_{1}, T_{1}\right),\left(\xi_{2}, T_{2}\right), \cdots,\left(\xi_{k}, T_{k}\right)\right\}$ is called a devision of $T$ and write

$$
\Pi=\left\{\left(\xi_{1}, T_{1}\right),\left(\xi_{2}, T_{2}\right), \cdots,\left(\xi_{k}, T_{k}\right)\right\} .
$$

For brevity, we write $\Pi=\{\xi$, $[u, v]\}$, where $[u, v]$ denotes a typical interval in $\Pi$ and $\xi$ is the associated point of $[u, v]$.

Definition 2.1 ([26]). Let $\delta(x)>0$ be a function on T. A division $\Pi$ of $T$ is said to be $\delta$-fine, if $\xi_{i} \in T_{i} \subset$ $\left(\xi_{i}-\delta\left(\xi_{i}\right), \xi_{i}+\delta\left(\xi_{i}\right)\right)(i=1,2, \cdots, k)$.

Definition 2.2 ([26]). A function $F: T \rightarrow R^{n}$ is said to be Henstock integrable on $T$ if for $A \in R^{n}$ and every $\varepsilon>0$, there is a function $\delta(x)>0$, such that for any $\delta$-fine devision $\Pi=\{\xi,[u, v]\}$ we have

$$
\left\|\sum_{(\Pi)} F(\xi)(v-u)-A\right\|<\varepsilon
$$

As usual, we write $(H) \int_{T} F(t) d t=A$. Here $\|\cdot\|$ stands for the norm on the $R^{n}$.

Throughout this paper, we use $P_{k}\left(R^{n}\right)$ to denote the family of all nonempty compact convex subsets of $R^{n}$. For $A, B \in P_{k}\left(R^{n}\right), k \in R$, the addition and scalar multiplication are defined by the equations as follows respectively:

$$
A+B=\{x+y \mid x \in A, y \in B\}, \quad a A=\{a x \mid x \in A\} .
$$


In addition, for $A, B \in P_{k}\left(R^{n}\right)$, the Hausdorff metric between them defined by

$$
d(A, B)=\max \left\{\sup _{a \in A} \inf _{b \in B}\|a-b\|, \sup _{b \in B} \inf _{a \in A}\|b-a\|\right\} .
$$

Definition 2.3. For $A \in P_{k}\left(R^{n}\right), x \in S^{n-1}$, the support function of $A$ is defined by

$$
\sigma(x, A)=\sup _{y \in A}\langle y, x\rangle
$$

where $S^{n-1}$ denotes the unit sphere of $R^{n},\langle\cdot, \cdot\rangle$ is the inner product in $R^{n}$.

It is clear that for $A, B \in P_{k}\left(R^{n}\right), x \in S^{n-1}$, we have

(1) $\sigma(x, k A)=k \sigma(x, A)(k \geqslant 0)$;

(2) $\sigma(x, A+B)=\sigma(x, A)+\sigma(x, B)$.

Theorem 2.4 ([12]). Let $A, B \in P_{k}\left(R^{n}\right)$. Then $d(A, B)=\sup _{x \in S^{n-1}}|\sigma(x, A)-\sigma(x, B)|$.

Definition $2.5([12,38])$. Let $E^{n}=\left\{u \mid u: R^{n} \rightarrow[0,1]\right\}$. For any $u \in E^{n}, u$ is said to be an $n$-dimensional fuzzy number if the following conditions are satisfied:

(1) $u$ is a normal fuzzy set, i.e., there exists an $x_{0} \in R^{n}$, such that $u\left(x_{0}\right)=1$;

(2) $u$ is a convex fuzzy set, i.e., $u(t x+(1-t) y) \geqslant \min \{u(x), u(y)\}$ for any $x, y \in R^{n}, t \in[0,1]$;

(3) $u$ is upper semi-continuous;

(4) suppu $=\overline{\left\{x \in R^{n} \mid u(x)>0\right\}}$ is compact, here $\bar{A}$ denotes the closure of $A$.

We define $D: E^{n} \times E^{n} \rightarrow[0, \infty)$ by the equation

$$
\mathrm{D}(\mathrm{u}, v)=\sup _{\mathrm{r} \in[0,1]} \mathrm{d}\left([\mathrm{u}]^{\mathrm{r}},[v]^{\mathrm{r}}\right), \quad \mathrm{u}, v \in \mathrm{E}^{\mathrm{n}},
$$

then the metric space $\left(E^{n}, D\right)$ has a linear structure, it can be imbedded isomorphically as a convex cone with vertex $\theta$ into the Banach space of functions $u^{*}: I \times S^{n-1} \longrightarrow R$, where $S^{n-1}$ is the unit sphere in $R^{n}$, with an imbedding function $u^{*}=j(u)$ defined by

$$
u^{*}(r, x)=\sup _{\alpha \in[u]^{\alpha}}<\alpha, x>
$$

for all $<r, x>\in \mathrm{I} \times \mathrm{S}^{\mathrm{n}-1}$

Theorem 2.6 ([38]). There exists a real Banach space $X$ such that $\mathrm{E}^{\mathrm{n}}$ can be imbedding as a convex cone $\mathrm{C}$ with vertex $\theta$ into X. Furthermore the following conditions hold true:

(1) the imbedding $\mathrm{j}$ is isometric;

(2) addition in $\mathrm{X}$ induces addition in $\mathrm{E}^{\mathrm{n}}$;

(3) multiplication by nonnegative real number in $\mathrm{X}$ induces the corresponding operation in $\mathrm{E}^{n}$;

(4) $\mathrm{C}-\mathrm{C}$ is dense in $\mathrm{X}$;

(5) $\mathrm{C}$ is closed. 
A fuzzy-number-valued function $\tilde{F}:[a, b] \rightarrow E^{n}$ is said to satisfy the condition $(H)$ on $[a, b]$, if for any $x_{1}<x_{2} \in[a, b]$ there exists $u \in E^{n}$ such that $f\left(x_{2}\right)=f\left(x_{1}\right)+u$. We call $u$ is the H-difference of $\tilde{F}\left(x_{2}\right)$ and $\tilde{\mathrm{F}}\left(x_{1}\right)$, denoted $\tilde{\mathrm{F}}\left(x_{2}\right)-_{\mathrm{H}} \tilde{\mathrm{F}}\left(\mathrm{x}_{1}\right)([22])$.

For brevity, we always assume that the condition $(\mathrm{H})$ is satisfied when dealing with the operation of subtraction of fuzzy numbers throughout this paper.

Definition $2.7([3])$. Let $\tilde{F}:(a, b) \rightarrow E^{n}$ and $x_{0} \in(a, b)$. We say that $\tilde{F}$ is differentiable at $x_{0}$, if there exists an element $\tilde{F}^{\prime}\left(t_{0}\right) \in E^{n}$, such that

(1) for all $h>0$ sufficiently small, there exist $\tilde{F}\left(x_{0}+h\right)-_{H} \tilde{F}\left(x_{0}\right), \tilde{F}\left(x_{0}\right)-{ }_{H} \tilde{F}\left(x_{0}-h\right)$ and the limits (in the metric D)

$$
\lim _{h \rightarrow 0} \frac{\tilde{F}\left(x_{0}+h\right)-_{H} \tilde{F}\left(x_{0}\right)}{h}=\lim _{h \rightarrow 0} \frac{\tilde{F}\left(x_{0}\right)-{ }_{H} \tilde{F}\left(x_{0}-h\right)}{h}=\tilde{F}^{\prime}\left(x_{0}\right),
$$

or;

(2) for all $h>0$ sufficiently small, there exist $\tilde{F}\left(x_{0}\right)-_{H} \tilde{F}\left(x_{0}+h\right), \tilde{F}\left(x_{0}-h\right)-_{H} \tilde{F}\left(x_{0}\right)$ and the limits

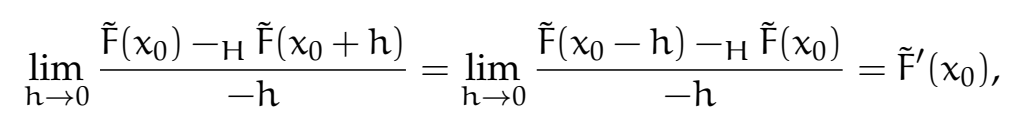

( $h$ and $-h$ at denominators mean $\frac{1}{h}$. and $-\frac{1}{h}$, respectively).

Theorem 2.8 ([12, 38]). If $u, v \in E^{n}, x, y \in S^{n-1}, r \in[0,1]$, then

(1) $\sigma\left(x+y,[u]^{r}\right) \leqslant \sigma\left(x,[u]^{r}\right)+\sigma\left(y,[u]^{r}\right)$;

(2) $\sigma\left(k x,[u]^{r}\right)=k \sigma\left(x,[u]^{r}\right)$ whenever $k \geqslant 0$;

(3) $\sigma\left(x,[u]^{r}\right)$ is bounded uniformly on $S^{n-1} \times I$, and $\sigma\left(x,[u]^{r}\right) \leqslant \sup _{a \in[u]^{0}}\|a\|$;

(4) $\sigma\left(x,[u]^{r}\right)$ is nonincreasing left continuous for any $x \in S^{n-1}$ with respect to $r$, especially it is right continuous at $\mathrm{r}=0$;

(5) $\sigma\left(x,[u]^{r}\right)$ is Lipschitz continuous uniformly for any $r \in[0,1]$ with respect to $x$, and

$$
\left|\sigma\left(x,[u]^{r}\right)-\sigma\left(y,[u]^{r}\right)\right| \leqslant\left(\sup _{a \in[u]^{r}}\|a\|\right)\|x-y\| ;
$$

(6) $\mathrm{d}\left([u]^{\mathrm{r}},[v]^{\mathrm{r}}\right)=\left|\sigma\left(x,[u]^{\mathrm{r}}\right)-\sigma\left(x,[v]^{\mathrm{r}}\right)\right|$.

In the following, we give the definition of Henstock-Pettis integral of fuzzy-number-valued functions and its representation theorems.

Definition $2.9([36,37])$. A fuzzy-number-valued function $\tilde{F}: T \rightarrow E^{n}$ is said to be fuzzy Henstock integrable on $T$ if there exists a fuzzy number $\tilde{A} \in E^{n}$ such that for every $\varepsilon>0$ there is a function $\delta(x)>0$ such that for any $\delta$-fine division $\Pi=\left\{\xi_{i},\left[x_{i-1}, x_{i}\right]\right\}$ of $\mathrm{T}$, we have

$$
\mathrm{D}\left(\tilde{\mathrm{A}}, \sum_{i} \tilde{\mathrm{F}}\left(\xi_{i}\right)\left(x_{i}-x_{i-1}\right)\right)<\varepsilon \text {. }
$$

We write $(\mathrm{FH}) \int_{\mathrm{T}} \tilde{\mathrm{F}}(\mathrm{x}) \mathrm{d} x=\tilde{\mathrm{A}}$.

Definition 2.10. A fuzzy-number-valued function $\tilde{F}: T \rightarrow E^{n}$ is said to be Henstock-Pettis integrable on $\mathrm{T}$ if $[\mathrm{F}(\mathrm{t})]^{r}$ is Henstock-Pettis integrable on $\mathrm{T}$ for every $r \in[0,1]$, and there exists a fuzzy number $\tilde{A} \in E^{n}$ such that for any $x \in S^{n-1}$ we have

$$
\left(\sigma\left(x,[A]^{r}\right)=(H) \int_{T} \sigma\left(x,[F(t)]^{r}\right) d t\right) .
$$

We write $\tilde{A}=(F H P) \int_{T} \tilde{F}(t) d t$. 
Remark 2.11. In particular, if $\tilde{F}$ is degenerated into $F: T \rightarrow R^{n}$ and $\tilde{A}$ is degenerated into $A \in R^{n}$, then

$$
\sigma\left(x,[A]^{r}\right)=<x, A>.
$$

Remark 2.12. When $n=1$, if the fuzzy-number-valued function $\tilde{\mathrm{F}}: \mathrm{T} \rightarrow \mathrm{E}^{1}$ is Kaleva integrable on $\mathrm{T}$ (refer to [36]), then $\tilde{F}$ is also Pettis integrable.

Similar to the methods of $[36,37]$, we easily obtain the following results.

Theorem 2.13. Suppose $\tilde{\mathrm{F}}, \tilde{\mathrm{G}}: \mathrm{T} \rightarrow \mathrm{E}^{\mathrm{n}}$ are fuzzy-number-valued functions on $\mathrm{T}$.

(1) If $\tilde{\mathrm{F}}, \tilde{\mathrm{G}}$ are fuzzy Henstock-Pettis integrable on $\mathrm{T}$, then $\alpha \tilde{\mathrm{F}}+\beta \tilde{\mathrm{G}}(\alpha, \beta \in \mathrm{R})$ is also fuzzy Henstock-Pettis integrable on $\mathrm{T}$, and

$$
(F H P) \int_{T}(\alpha \tilde{F}(t)+\beta \tilde{G}(t)) d t=\alpha(F H P) \int_{T} \tilde{F}(t) d t+\beta(F H P) \int_{T} \tilde{G}(t) d t .
$$

(2) If $\tilde{\mathrm{F}}$ is fuzzy Henstock-Pettis integrable on $\mathrm{T}$, then $\tilde{\mathrm{F}}$ is fuzzy Henstock-Pettis integrable on every subset of $\mathrm{T}$, and for nonoverlapping $\mathrm{T}_{1}, \mathrm{~T}_{2}, \cdots, \mathrm{T}_{\mathrm{m}}$ we have

$$
(F H P) \int_{T} \tilde{F}(t) d t=\sum_{i=1}^{m}(F H P) \int_{T_{i}} \tilde{F}(t) d t,
$$

where $\mathrm{T}=\bigcup_{i=1}^{m} \mathrm{~T}_{i}$.

(3) If $\tilde{\mathrm{F}}$ is fuzzy Henstock-Pettis integrable on $\mathrm{T}$ and $\tilde{\mathrm{F}}=\tilde{\mathrm{G}}$ almost everywhere on $\mathrm{T}$, then $\tilde{\mathrm{G}}$ is also fuzzy Henstock-Pettis integrable on $\mathrm{T}$ and

$$
(F H P) \int_{T} \tilde{F}(t) d t=(F H P) \int_{T} \tilde{G}(t) d t
$$

Theorem 2.14. A fuzzy-number-valued function $\tilde{\mathrm{F}}: \mathrm{T} \rightarrow \mathrm{E}^{\mathrm{n}}$ is fuzzy Henstock-Pettis integrable on $\mathrm{T}$ if and only if for every $r \in[0,1]$, real-valued function $\sigma\left(x,[\mathrm{~F}(\mathrm{t})]^{\mathrm{r}}\right)$ is Henstock integrable uniformly on $\mathrm{T}$ for any $\mathrm{x} \in \mathrm{S}^{\mathrm{n}-1}$, and

$$
\sigma\left(x,(H) \int_{T}[F(t)]^{r} d t\right)=(H) \int_{T} \sigma\left(x,[F(t)]^{r}\right) d t .
$$

Theorem 2.15. If fuzzy-number-valued function $\tilde{\mathrm{G}}: \mathrm{T} \rightarrow \mathrm{E}^{\mathrm{n}}$ is Pettis integrable on $\mathrm{T}$ and the null function is a selection of $[\mathrm{G}(\mathrm{t})]^{\mathrm{r}}, \mathrm{r} \in[0,1]$, then for every $\mathrm{A}, \mathrm{B} \subset \mathrm{T}$ such that $\mathrm{A} \subseteq \mathrm{B}$ we have $w_{\mathrm{A}} \leqslant w_{\mathrm{B}}$, where $w_{\mathrm{A}}=(\mathrm{FP}) \int_{\mathrm{A}} \tilde{\mathrm{G}}(\mathrm{t}) \mathrm{dt}, w_{\mathrm{B}}=(\mathrm{FP}) \int_{\mathrm{B}} \tilde{\mathrm{G}}(\mathrm{t}) \mathrm{dt}$.

Proof. Since $A, B \subset T, A \subseteq B$ and $[G(t)]^{r}: T \rightarrow P_{k}\left(R^{n}\right)$ is Pettis integrable for any $r \in[0,1]$, we have $\left[w_{A}\right]^{r} \subset\left[w_{B}\right]^{r}$, i.e., $w_{A} \leqslant w_{B}$, where

$$
\left[w_{A}\right]^{r}=(P) \int_{A}[G(t)]^{r} d t, \quad\left[w_{B}\right]^{r}=(P) \int_{B}[G(t)]^{r} d t
$$

Example 2.16. We present an example of function which is fuzzy Henstock-Pettis integrable and neither strong fuzzy Henstock integrable nor Keleva integrable.

Let $\tilde{f}:[0,1] \rightarrow E^{n}$ and let $\tilde{f}(t)=\chi_{\left\{\tilde{F}^{\prime}(t)\right\}}+\tilde{A}(s) \cdot \tilde{F}^{\prime}(t)$, where

$$
\tilde{F}(t)= \begin{cases}t^{2} \sin t^{-2}, & t \neq 0 \\ 0, & t=0\end{cases}
$$


and

$$
\tilde{A}(s)= \begin{cases}s, & 0 \leqslant s \leqslant 1, \\ 2-s, & 1<s \leqslant 2, \\ 0, & \text { others. }\end{cases}
$$

Put $\tilde{f}_{1}(t)=\chi_{\left\{\tilde{F}^{\prime}(t)\right\}}$ and $\tilde{f}_{2}=\tilde{A}(s) \cdot \tilde{F}^{\prime}(t)$. We can prove that a function $\tilde{f}=\tilde{f}_{1}+\tilde{f}_{2}$ is integrable in the sense of fuzzy Henstock-Pettis. In fact, $\sigma\left(x,[f(t)]^{r}\right)$ is Henstock integrable on $[0,1]$. In addition, the function $\tilde{f}$ is not Keleva integrable because $j \circ \tilde{f}_{2}$ is not Lebesgue integrable. Moreover, $\tilde{f}_{1}$ is not strong measurable, so this function is not strong fuzzy Henstock integrable.

\section{The existence of generalized weak solutions to discontinuous fuzzy differential equations}

Convergence theorems for a given integration theory are important for estimating the power of the theory. For real and Banach-valued Henstock integrable functions, there are a few convergence theorems (see $[26,29]$ for instance). In order to generalize certain results on continuous dependence of solutions of ordinary differential equations with respect to parameters, Kurzweil introduced, in 1957, what he called generalized ordinary differential equations for Euclidean and Banach space-valued functions (see [25]). The theory of generalized ordinary differential equations is extensively described in [28]. In [10] and [11], the authors extended the controlled convergence theorems and proved the existence theorems for the Cauchy problem for Banach space-valued functions under Henstock-Pettis integrability assumptions, respectively. In this section, we present a controlled convergence theorem for fuzzy Henstock-Pettis integral. At last, we also give an existence theorem for a Cauchy problem using the fuzzy Henstock-Pettis integral and its properties. The requirements on the right hands function $\tilde{f}$ are not too restrictive.

Definition 3.1. Let $X \subset[a, b]$. An $n$-dimensional fuzzy-number-valued function $\tilde{F}$ defined on $X$ is said to be $A C(X)$ if for every $\varepsilon>0$ such that for every finite sequence of non-overlapping intervals $\left\{\left[a_{i}, b_{i}\right]\right\}$, with $\sum_{i=1}^{n}\left|b_{i}-a_{i}\right|<\eta$, we have

$$
\sum \mathrm{D}\left(\tilde{\mathrm{F}}\left(\mathrm{b}_{i}\right), \tilde{\mathrm{F}}\left(\mathrm{a}_{i}\right)\right)<\varepsilon
$$

where the endpoints $a_{i}, b_{i} \in X$ for all $i$.

Definition 3.2. An $n$-dimensional fuzzy-number-valued function $\tilde{F}$ defined on $X \subset[a, b]$ is said to be uniformly $A C^{*}(X)$ if for every $\varepsilon>0$ there exists $\eta>0$ such that for every finite sequence of nonoverlapping intervals $\left\{\left[a_{i}, b_{i}\right]\right\}$, satisfying $\sum_{i=1}^{n}\left|b_{i}-a_{i}\right|<\eta$ where $a_{i}, b_{i} \in X$ for all $i$, we have

$$
\sum \omega\left(\tilde{\mathrm{F}},\left[\mathrm{a}_{i}, \mathrm{~b}_{i}\right]\right)<\varepsilon
$$

where $\omega$ denotes the oscillation of $\tilde{F}$ over $\left[a_{i}, b_{i}\right]$, i.e.,

$$
\omega\left(\tilde{F},\left[a_{i}, b_{i}\right]\right)=\sup \left\{D(\tilde{F}(y), \tilde{F}(x)): x, y \in\left[a_{i}, b_{i}\right]\right\} .
$$

Definition 3.3. An $n$-dimensional fuzzy-number-valued function $\tilde{F}$ is said to be $A C G^{*}$ on $X$, if $X$ is the union of a sequence of closed sets $\left\{X_{i}\right\}$ such that on each $X_{i}, \tilde{F}$ is $A C^{*}\left(X_{i}\right)$.

An $n$-dimensional fuzzy-number-valued function $\tilde{F}$ is said to be uniformly $A_{C G}{ }^{*}$ on $X$, if $X$ is the union of a sequence of closed sets $\left\{X_{i}\right\}$ such that on each $X_{i}, \tilde{F}$ is uniformly $A C^{*}\left(X_{i}\right)$.

Theorem 3.4 (Controlled Convergence Theorem). Let $\tilde{f_{n}}, \tilde{f}: T \rightarrow E^{n}$ be (FHP)-integrable functions on $T$. Assume that

(1) $\sigma\left(x,\left[f_{n}(t)\right]^{r}\right) \longrightarrow \sigma\left(x,[f(t)]^{r}\right)$, a.e. on $T$;

(2) the family $\mathrm{G}=\left\{\sigma\left(\mathrm{x},\left[\mathrm{F}_{\mathrm{n}}(\mathrm{t})\right]^{\mathrm{r}}\right), \mathrm{n}=1,2, \cdots\right\}$ is uniformly $\mathrm{ACG}^{*}$ and equicontinuous on $\mathrm{T}$. 
Then $\tilde{\mathrm{f}}$ is (FHP)-integrable and

$$
\lim _{n \rightarrow \infty}(F H P) \int_{0}^{t} \tilde{f_{n}}(s) d s=(F H P) \int_{0}^{t} \tilde{f}(s) d s
$$

Proof.

(i) Since $\tilde{f_{n}}$ is (FHP)-integrable, the function $\sigma\left(x,\left[f_{n}(t)\right]^{r}\right)$ is $(H)$-integrable. So,

(a) $\sigma\left(x,\left[f_{n}(t)\right]^{r}\right) \longrightarrow \sigma\left(x,[f(t)]^{r}\right)$, a.e. on $T$;

(b) G is uniformly ACG*;

(c) the sequence $\sigma\left(x,\left[F_{n}(t)\right]^{r}\right)$ is uniformly convergent on $T$.

By using the convergence theorem for Henstock integral [26], we have

$$
(\mathrm{H}) \int_{0}^{t} \sigma\left(x,\left[f_{n}(s)\right]^{r}\right) \mathrm{d} s \longrightarrow(H) \int_{0}^{t} \sigma\left(x,[f(s)]^{r}\right) d s .
$$

(ii) Fix an arbitrary $t \in T$, and let $\tilde{g_{n}} \subset \tilde{f_{n}}$. For every $\varepsilon>0$ there exists $\sigma^{*}$ such that

$$
\sigma^{*}\left(x,\left[g_{n}(s)\right]^{r}\right) \subset \sigma\left(x,\left[f_{n}(s)\right]^{r}\right),
$$

with following conditions:

$$
\lim _{n \rightarrow \infty}(H) \int_{0}^{t} \sigma^{*}\left(x,\left[g_{n}(s)\right]^{r}\right) d s=(H) \int_{0}^{t} \sigma\left(x,[f(s)]^{r}\right) d s .
$$

Consider the set $\left\{\sigma^{*}\left(x,\left[f_{n}(t)\right]^{r}\right) \mid n=1,2, \cdots\right\}$, there exists a subsequence $\sigma^{*}\left(x,\left[g_{n}(s)\right]^{r}\right) \subset \sigma\left(x,\left[f_{n}(s)\right]^{r}\right)$ such that the limit $\lim _{k \rightarrow \infty} \sigma_{k}^{*}\left(x,[f(t)]^{r}\right)$ exists almost everywhere and

$$
\lim _{k \rightarrow \infty} \sigma_{k}^{*}\left(x,[f(t)]^{r}\right)=\sigma_{0}^{*}\left(x,[f(t)]^{r}\right) .
$$

Since $\sigma\left(x,\left[f_{m}(s)\right]^{r}\right)$ is uniformly $(H)$-integrable, that is, for all $\varepsilon>0$, there exists a $\delta$-fine partition on $T$ such that

$$
\left|\sum_{j=1}^{k} \sigma\left(x,\left[f_{m}\left(t_{j}\right)\right]^{r} \cdot\left(x_{i}-x_{j-1}\right)\right)-(H) \int_{a}^{b} \sigma\left(x,\left[f_{m}(s)\right]^{r}\right) d s\right|<\varepsilon .
$$

By (3.1) and (3.2), we have

$$
\begin{aligned}
\left|\sum_{j=1}^{k} \sigma_{k}^{*}\left(x,\left[f_{m}\left(t_{j}\right)\right]^{r} \cdot\left(x_{i}-x_{j-1}\right)\right)-(H) \int_{a}^{b} \sigma_{k}^{*}\left(x,[f(s)]^{r}\right) d s\right| \\
\leqslant \mid \\
\quad\left|\sum_{j=1}^{k} \sigma_{k}^{*}\left(x,\left[f_{m}\left(t_{j}\right)\right]^{r} \cdot\left(x_{i}-x_{j-1}\right)\right)-\sum_{j=1}^{k} \sigma_{k}^{*}\left(x,\left[g_{m}\left(t_{j}\right)\right]^{r} \cdot\left(x_{i}-x_{j-1}\right)\right)\right| \\
\quad+\left|\sum_{j=1}^{k} \sigma_{k}^{*}\left(x,\left[g_{m}\left(t_{j}\right)\right]^{r} \cdot\left(x_{i}-x_{j-1}\right)\right)-(H) \int_{a}^{b} \sigma_{k}^{*}\left(x,[f(s)]^{r}\right) d s\right| \\
\quad+\left|(H) \int_{a}^{b} \sigma_{k}^{*}\left(x,[f(s)]^{r}\right) d s-(H) \int_{a}^{b} \sigma_{k}^{*}\left(x,[f(s)]^{r}\right) d s\right| .
\end{aligned}
$$

By condition (1), there exists $m_{0} \in \mathbb{N}$ such that $m \geqslant m_{0}$ and we have

$$
\left|\sum_{j=1}^{k} \sigma_{k}^{*}\left(x,\left[f_{m}\left(t_{j}\right)\right]^{r} \cdot\left(x_{i}-x_{j-1}\right)\right)-\sum_{j=1}^{k} \sigma_{k}^{*}\left(x,\left[g_{m}\left(t_{j}\right)\right]^{r} \cdot\left(x_{i}-x_{j-1}\right)\right)\right|<\frac{\varepsilon}{3} .
$$


According the uniform $(H)$-integrability of $\sigma^{*}\left(x,\left[f_{m}(s)\right]^{r}\right)$, we have

$$
\left|\sum_{j=1}^{k} \sigma_{k}^{*}\left(x,\left[g_{m}\left(t_{j}\right)\right]^{r} \cdot\left(x_{i}-x_{j-1}\right)\right)-(H) \int_{a}^{b} \sigma_{k}^{*}\left(x,[f(s)]^{r}\right) d s\right|<\frac{\varepsilon}{3} .
$$

By (i),

$$
\left|(H) \int_{a}^{b} \sigma_{k}^{*}\left(x,[f(s)]^{r}\right) d s-(H) \int_{a}^{b} \sigma_{k}^{*}\left(x,[f(s)]^{r}\right) d s\right|<\frac{\varepsilon}{3} .
$$

So the set $\left\{\sigma^{*}\left(x,\left[f_{n}(t)\right]^{r}\right) \mid n=1,2, \cdots\right\}$ is uniformly integrable.

Now we are able to use the Vitali convergence theorem for real-valued Henstock integrable functions [26] and see that

$$
\lim _{k \rightarrow \infty}(H) \int_{0}^{t} \sigma_{k}^{*}\left(x,[f(t)]^{r}\right) d s=(H) \int_{0}^{t} \sigma_{0}^{*}\left(x,[f(s)]^{r}\right) d s .
$$

From (3.2), we get that

$$
\lim _{k \rightarrow \infty}(H) \int_{0}^{t} \sigma_{k}^{*}\left(x,\left[g_{n}(s)\right]^{r}\right) d s=(H) \int_{0}^{t} \sigma_{0}^{*}\left(x,[f(s)]^{r}\right) d s .
$$

Thus, for all $n>k$, we have

$$
(H) \int_{0}^{t} \sigma_{k}^{*}\left(x,\left[g_{n}(s)\right]^{r}\right) d s>\varepsilon
$$

and

$$
(H) \int_{0}^{t} \sigma_{k}^{*}\left(x,[f(s)]^{r}\right) d s \geqslant \varepsilon
$$

for all $k=1,2, \cdots$. Passing to the limit with $k \rightarrow \infty$,

$$
(H) \int_{0}^{t} \sigma_{0}^{*}\left(x,[f(s)]^{r}\right) d s \geqslant \varepsilon .
$$

Since $\sigma_{\alpha}^{*}\left(x,\left[g_{n}(s)\right]^{r}\right)$ is uniformly convergent to $\sigma_{\alpha}^{*}\left(x,[g(s)]^{r}\right)$ for $n$, we have

$$
\lim _{\alpha}(H) \int_{0}^{t} \sigma_{\alpha}^{*}\left(x,\left[g_{n}(s)\right]^{r}\right) d s=\lim _{\alpha} \sigma\left(x,\left[(F H P) \int_{0}^{t} \tilde{g_{n}}(s) d s\right]^{r}\right) .
$$

Denote $\tilde{z_{\mathfrak{n}}}(\mathrm{t})=(\mathrm{FHP}) \int_{0}^{\mathrm{t}} \tilde{\mathrm{g}_{\mathrm{n}}}(\mathrm{s}) \mathrm{d}$ s. In this case, we see that

$$
\begin{aligned}
\lim _{\alpha} \int_{0}^{t} \sigma_{\alpha}^{*}\left(x,\left[g_{n}(s)\right]^{r}\right) \mathrm{d} s & =\lim _{\alpha} \sigma\left(x,\left[z_{\mathfrak{n}}(t)\right]^{r}\right) \\
& =\sigma_{0}^{*}\left(x,\left[(F H P) \int_{0}^{t} \tilde{g_{n}}(s) d s\right]^{r}\right) \\
& =(H) \int_{0}^{t} \sigma_{0}^{*}\left(x,\left[g_{\mathfrak{n}}(s)\right]^{r}\right) d s .
\end{aligned}
$$

From (3.3), we have $\int_{0}^{t} \sigma_{0}^{*}\left(x,[f(s)]^{r}\right) d s=0$ which contradicts (3.4). Since

$$
(\mathrm{H}) \int_{0}^{t} \sigma\left(x,\left[f_{n}(s)\right]^{r}\right) \mathrm{d} s \longrightarrow(H) \int_{0}^{t} \sigma\left(x,[f(s)]^{r}\right) d s,
$$

and according to (i), a sequence $\left\{(\mathrm{FHP}) \int_{0}^{\mathrm{t}} \tilde{\mathrm{f}_{\mathrm{n}}}(\mathrm{s}) \mathrm{d} s\right\}$ is Cauchy, so

$$
\lim _{n \rightarrow \infty}(F H P) \int_{0}^{t} \tilde{f_{n}}(s) d s=(F H P) \int_{0}^{t} \tilde{f}(s) d s .
$$


Since the convergence theorems are really applicable (fuzzy differential equations, for instance), we shall present a few particular case of Theorem 3.4.

Corollary 3.5. If condition (2) is replaced by condition:

(3) for each $i, k \in \mathbb{N}$ there exists real-valued Henstock integrable function $\mathrm{g}: \mathrm{T} \longrightarrow \mathrm{R}$, such that

$$
\left|\sigma\left(x,\left[f_{i}(t)\right]^{r}\right)-\sigma\left(x,\left[f_{k}(t)\right]^{r}\right)\right| \leqslant g .
$$

Then the conclusion of Theorem 3.4 also holds.

Corollary 3.6. If condition (2) is replaced by condition:

(4) for each $\mathrm{n} \in \mathbb{N}$ there exist real-valued Henstock integrable functions $\mathrm{g}, \mathrm{h}: \mathrm{T} \longrightarrow \mathrm{R}$, such that

$$
g \leqslant \sigma\left(x,\left[f_{n}(t)\right]^{r}\right) \leqslant h ;
$$

then the conclusion of Theorem 3.4 also holds.

Next, we will deal with the Cauchy problem:

$$
\left\{\begin{array}{l}
x^{\prime}(t)=\tilde{f}(t, x(t)), \quad t \in[0, \alpha]=I_{\alpha} \\
x(0)=x_{0}
\end{array}\right.
$$

where $\tilde{f}$ is fuzzy Henstock-Pettis integrable function. In fact, our existence theorem is based on an idea of Kurzweil from [25].

Definition 3.7. Let $\tilde{F}: T \rightarrow E^{n}$ be fuzzy-number-valued function and let $A \subset T$. The function $f: A \rightarrow E^{n}$ is the weak derivative of $F$ on $A$, if the Banach-valued function $j \circ \tilde{F}$ is differentiable almost everywhere on $A$ and $(j \circ \tilde{F})^{\prime}=j \circ \tilde{f}$, a.e. .

Definition 3.8. A fuzzy-number-valued function $\tilde{f}: I_{\alpha} \longrightarrow E^{n}$ is weak continuous if $j \circ \tilde{f}$ is continuous on $\mathrm{I}_{\alpha}$.

Theorem 3.9. Let $\tilde{f}:[a, b] \longrightarrow E^{n}$ be (FHP)-integrable on $[a, b]$ and let $\tilde{F}(x)=\int_{a}^{x} \tilde{f}(s) d s$. Then

(1) $\sigma\left(x,[f(s)]^{r}\right)$ is Henstock integrable on $[a, b]$ and $(H) \int_{a}^{x} \sigma\left(x,[f(s)]^{r}\right) d s=\sigma\left(x,[F(x)]^{r}\right)$;

(2) the function $\tilde{\mathrm{F}}$ is weak continuous on $[\mathrm{a}, \mathrm{b}]$ and $\tilde{\mathrm{f}}$ is a weak derivative of $\tilde{\mathrm{F}}$ on $[\mathrm{a}, \mathrm{b}]$.

Proof.

(1) See Definition 2.10.

(2) Since the function $\sigma\left(x,[f(s)]^{r}\right)$ is a real-valued and $(H)$-integrable, and

$$
(H) \int_{a}^{x} \sigma\left(x,[f(s)]^{r}\right) d s=\sigma\left(x,[F(x)]^{r}\right),
$$

then $G(x)=\int_{a}^{x} \tilde{f}(s) d s$ is continuous, that is $\tilde{F}$ is weak continuous. By conclusion (a), there exists a set $A \subset[a, b]$, such that $G^{\prime}(x)=\sigma\left(x,[f(s)]^{r}\right)$, but $G^{\prime}(x)=\sigma\left(x,[F(x)]^{r}\right)^{\prime}(x)$.

Definition 3.10. A function $x: I_{\alpha} \longrightarrow E^{n}$ is said to be a weak solution of Cauchy problem (3.5) if it satisfies the following conditions:

(1) $\chi(\cdot)$ is ACG $^{*}$;

(2) $x(0)=x_{0}$; 
(3) there exists a set $A$, with Lebesgue measure zero, such that for each $t \notin A$

$$
j \circ\left(x^{\prime}(t)\right)=j \circ(\tilde{f}(t, x(t))),
$$

where "ı" denotes the weak derivative.

Theorem 3.11. If the function $\tilde{\mathrm{f}}: \mathrm{I}_{\alpha} \longrightarrow \mathrm{E}^{\mathrm{n}}$ is (FHP)-integrable, then

$$
\int_{I} \tilde{f}(t) d t \in|I| \cdot \overline{\operatorname{conv}} \tilde{f}(I)
$$

where $\mathrm{I} \subset \mathrm{I}_{\alpha}$ and $|\mathrm{I}|$ is the lenght of $\mathrm{I}$.

Proof. Since $\sigma\left(x,[f(t)]^{r}\right)$ is $(H)$-integrable, by the mean value theorem for $\mathrm{H}$ integral we have

$$
(H) \int_{I} \sigma\left(x,[f(t)]^{r}\right) d t \in|I| \cdot \overline{\operatorname{conv}} \sigma\left(x,[f(I)]^{r}\right)=\sigma\left(x,|I| \cdot \overline{\operatorname{conv}}[f(I)]^{r}\right) .
$$

However, by the definition of fuzzy Henstock-Pettis integral, there exists

$$
\text { (H) } \int_{I} \sigma\left(x,[f(t)]^{r}\right) d t=\sigma\left(x,\left[\int_{I} \tilde{f}(t) d t\right]^{r}\right) .
$$

Therefore, $\sigma\left(x,\left[\int_{I} \tilde{f}(t) d t\right]^{r}\right) \in \sigma\left(x,|I| \cdot \overline{\operatorname{conv}}[f(I)]^{r}\right)$. Since $|I| \cdot \overline{\operatorname{conv}} \tilde{f}(I)$ is a closed convex set, we have

$$
\int_{I} \tilde{f}(t) d t \in|I| \cdot \overline{\operatorname{conv}} \tilde{f}(I) .
$$

Theorem 3.12 ([24]). Let $\mathcal{D}$ be a closed convex subset of a Banach space $\mathrm{X}$, and let $\mathrm{F}$ be a weakly sequentially continuous map of $\mathcal{D}$ into itself. If for some $x \in \mathcal{D}$ the implication

$$
\overline{\mathrm{V}}=\overline{\mathrm{conv}}(\{\mathrm{x}\} \cup \mathrm{F}(\mathrm{V})) \Longrightarrow \mathrm{V} \text { is relatively weakly compact, }
$$

holds for every subset $\mathrm{V}$ of $\mathcal{D}$, then $\mathrm{F}$ has a fixed point.

For any bounded subset $A$ of Banach space $X$ we denote $\mu(A)$ the measure of weak non-compactness of $A$, i.e.,

$$
\mu(A)=\inf \left\{t>0: \text { there exists } C \in \mathcal{K} \text { such that } A \subset C+t B_{0}\right\},
$$

where $\mathcal{K}$ is the set of weakly compact subsets of $X$ and $B_{0}$ is the norm unit ball in $X$. For the properties of the weak non-compactness $\mu(\cdot)$, we refer to [1] for example.

Lemma 3.13 ([1]). Let $\mathrm{H} \subset \mathrm{C}\left(\mathrm{I}_{\alpha}, \mathrm{X}\right)$ be a family of strong equicontinuous functions. Then $\mu\left(\mathrm{H}\left(\mathrm{I}_{\alpha}\right)\right)=\sup \mu(\mathrm{H}(\mathrm{t}))$ and the function $\mathrm{t} \rightarrow \mu(\mathrm{H}(\mathrm{t}))$ is continuous.

Let a closed and convex $C\left(x_{0}, \alpha\right)=\left\{x \in C\left(I_{\alpha}, E^{n}\right) \mid x(0)=x_{0}, D(x, \tilde{0}) \leqslant D\left(x_{0}, \tilde{0}\right)+b\right\}$ and let the sequence of functions $G=\left\{\tilde{F}_{x} \mid x \in C\left(x_{0}, \alpha\right)\right\}$. Define the operator $\tilde{F}_{x}$ by the following:

$$
\tilde{\mathrm{F}}_{x}(\mathrm{t})=x_{0}+\int_{0}^{t} \tilde{f}(s, x(s)) \mathrm{d} s \quad \text { or } \quad \tilde{\mathrm{F}}_{x}(\mathrm{t})=\mathrm{x}_{0}+(-1) \cdot \int_{0}^{t} \tilde{f}(s, x(s)) \mathrm{d} s
$$

for $\mathrm{t} \in \mathrm{I}_{\alpha}, x \in \mathrm{C}\left(\mathrm{x}_{0}, \alpha\right)$.

Theorem 3.14. Suppose that a function $x: \mathrm{I}_{\alpha} \longrightarrow \mathrm{E}^{\mathrm{n}}$ is $\mathrm{ACG}^{*} . \tilde{\mathrm{f}}(\cdot, x(\cdot))$ is (FHP)-integrable and $\tilde{\mathrm{f}}(\mathrm{t}, \cdot)$ is weak continuous about first variable $\mathrm{t}$ and

$$
\mu\left(\sigma\left(x,[\tilde{f}(I \times X)]^{r}\right)\right) \leqslant c \cdot \mu(\sigma(x, X)), \quad 0 \leqslant c \cdot \alpha<1
$$

for each bounded subset $\mathrm{X} \subset \mathrm{E}^{\mathrm{n}}$ and for each subinterval $\mathrm{I}$ of $\mathrm{I}_{\alpha}$. Assume that the set $\mathrm{G}=\left\{\tilde{\mathrm{F}}_{\mathrm{x}} \mid \mathrm{x} \in \mathrm{C}\left(\mathrm{x}_{0}, \alpha\right)\right\}$ is 
strong equicontinuous and weak uniformly $\mathrm{ACG}^{*}$ on $\mathrm{I}_{\alpha}$. Then there exists at least one weak solution of problem (3.5) on $\mathrm{I}_{\beta}$, for some number $0<\beta \leqslant \alpha$.

Proof. We will prove, in fact, the existence of a solution for the following problem:

$$
x(t)=x_{0}+\int_{0}^{t} \tilde{f}(s, x(s)) d s, \quad t \in I_{\alpha} .
$$

By Theorem 3.9 each solution of problem (3.8) is a solution of problem (3.5). Fix an arbitrary $b \geqslant 0$. By the equicontinuity of $G$, there exists a number $\beta, 0<\beta \leqslant \alpha$, such that

$$
D\left(\int_{0}^{t} \tilde{f}(s, x(s)) d s, \tilde{0}\right) \leqslant b
$$

for all $t \in \mathrm{I}_{\alpha}$ and $x \in \mathrm{C}\left(\mathrm{x}_{0}, \alpha\right)$.

Next, we prove $\tilde{F}$ is sequentially continuous. In fact, for every $t \in I_{\beta}$, there exist a sequence $x_{n}(t)$ convergent to $x(t)$ on $C\left(I_{\beta}, E^{n}\right)$. That is $\tilde{f}\left(t, x_{n}(t)\right) \longrightarrow \tilde{f}(t, x(t))$. By the Controlled Convergence Theorem 3.4, we have

$$
\lim _{n \rightarrow \infty} \int_{0}^{t} \tilde{f}\left(s, x_{n}(s)\right) d s=\int_{0}^{t} \tilde{f}(s, x(s)) d s .
$$

So, $\tilde{\mathrm{F}}_{x_{n}} \longrightarrow \tilde{\mathrm{F}}_{x}$. That is to say $\tilde{\mathrm{F}}$ is continuous.

Assume that $V \subset C\left(x_{0}, \beta\right)$ satisfies the condition $\bar{V}=\overline{\operatorname{conv}}(\tilde{F}(V) \cup\{x\})$. we shall prove $V$ is relatively compact, thus (3.6) is satisfied. In fact, let

$$
\tilde{\mathrm{F}}(\mathrm{V}(\mathrm{t}))=\left\{\tilde{\mathrm{F}}_{x} \mid x \in \mathrm{V}\right\}=\left\{x_{0}+\int_{0}^{\mathrm{t}} \tilde{\mathrm{f}}(\mathrm{s}, x(\mathrm{~s})) \mathrm{d} s, x \in \mathrm{V}\right\}
$$

By properties of the measure of weak non-compactness and the assumption (3.7), we have

$$
\begin{aligned}
\mu\left(\sigma\left(x,\left[\tilde{\mathrm{f}}(\mathrm{V}(\mathrm{t})]^{\mathrm{r}}\right)\right)\right. & =\mu\left(\sigma\left(x,\left[x_{0}+\int_{0}^{\mathrm{t}} \tilde{\mathrm{f}}(\mathrm{s}, \mathrm{x}(\mathrm{s})) \mathrm{d} s\right]^{\mathrm{r}}\right)\right) \\
& \leqslant \mu\left(\sigma\left(x,\left[\int_{0}^{\mathrm{t}} \tilde{\mathrm{f}}(\mathrm{s}, \mathrm{x}(\mathrm{s})) \mathrm{ds}\right]^{\mathrm{r}}\right)\right) \\
& \leqslant \mu\left(\sigma\left(x, \mathrm{t} \cdot \overline{\operatorname{conv}}[\tilde{\mathrm{f}}([0, \mathrm{t}] \times \mathrm{V}([0, \mathrm{t}]))]^{\mathrm{r}}\right)\right) \\
& \leqslant \mathrm{t} \cdot \mu\left(\sigma\left(x,[\tilde{\mathrm{f}}([0, \mathrm{t}] \times \mathrm{V}([0, \mathrm{t}]))]^{\mathrm{r}}\right)\right) \\
& \leqslant \beta \cdot \mu\left(\sigma\left(x,\left[\tilde{\mathrm{f}}\left(\mathrm{I}_{\beta} \times \mathrm{V}\left(\mathrm{I}_{\beta}\right)\right)\right]^{\mathrm{r}}\right)\right) \\
& \leqslant \beta \cdot c \cdot \mu\left(\sigma\left(x, \mathrm{~V}\left(\mathrm{I}_{\beta}\right)\right)\right) .
\end{aligned}
$$

Hence $\mu\left(\sigma\left(x,[\tilde{F}(V(t))]^{r}\right)\right) \leqslant \beta \cdot c \cdot \mu\left(\sigma\left(x, V\left(I_{\beta}\right)\right)\right)$ for each $t \in I_{\beta}$.

Because $V=\overline{\operatorname{conv}}(\tilde{\mathrm{F}}(\mathrm{V}) \cup\{x\})$ then

$$
\mu(\sigma(x, V(t))) \leqslant \mu\left(\sigma\left(x,[\tilde{F}(V(t))]^{r}\right)\right) \leqslant \beta \cdot c \cdot \mu\left(\sigma\left(x, V\left(I_{\beta}\right)\right)\right) .
$$

By Lemma 3.13, we have

$$
\mu\left(\sigma\left(x, V\left(I_{\beta}\right)\right)\right) \leqslant \beta \cdot c \cdot \mu\left(\sigma\left(x, V\left(I_{\beta}\right)\right)\right) \leqslant \alpha \cdot c \cdot \mu\left(\sigma\left(x, V\left(I_{\beta}\right)\right)\right) .
$$

So, $\mu\left(\sigma\left(x, V\left(I_{\beta}\right)\right)\right)=0$ and $\mu(\sigma(x, V(t)))=0$ for each $t \in I_{\beta}$. By Arzelá-Ascoli theorem $V$ is relatively compact in $C\left(I_{\beta}, E^{n}\right)$. Using Theorem 3.12 there exists a fixed point of the operator $F$ which is a weak solution of problem (3.5).

Example 3.15. Consider the following discontinuous system

$$
\left\{\begin{array}{l}
\tilde{x}^{\prime}(t)=\tilde{f}(t, \tilde{x})+\tilde{h}(t), \\
\tilde{x}(0)=\tilde{x_{0}},
\end{array}\right.
$$


where $\tilde{f}(t, \tilde{x})$ is (FHP)-integrable and $\tilde{f}(t, \cdot)$ is weak continuous about first variable $t, h(t)=\chi_{\{g(t)\}}+\tilde{A}$ is a fuzzy-number-valued function and

$$
g(t)= \begin{cases}2 t \sin \frac{1}{t^{2}}-\frac{2}{t} \cos \frac{1}{t^{2},} & t \neq 0, \\ 0, & t=0,\end{cases}
$$

and

$$
\tilde{A}(s)= \begin{cases}s, & 0 \leqslant s \leqslant 1, \\ 2-s, & 1<s \leqslant 2, \\ 0, & \text { others. }\end{cases}
$$

Since $\tilde{x}(t)$ is a generalized solution of the initial value problem (3.9) if and only if the integral equation

$$
\tilde{x}(t)=\tilde{x}_{0}+(F H P) \int_{0}^{t}(\tilde{f}(s, \tilde{x}(s))+\tilde{h}(s)) d s,
$$

holds true. That is to say

$$
\tilde{x}(t)=\tilde{H}(t)+(F H P) \int_{0}^{t} \tilde{f}(s, \tilde{x}(s)) d s,
$$

where $\tilde{\mathrm{H}}=\tilde{\chi_{0}}+\chi_{\{G(t)\}}+\tilde{A} \cdot t$ and

$$
G(t)= \begin{cases}t^{2} \sin \frac{1}{t^{2}}, & t \neq 0 \\ 0, & t=0\end{cases}
$$

Let $\tilde{f}(s, \tilde{x})$ to be satisfied (3.7) in Theorem 3.14 and $H=\left\{\tilde{H}_{x} \mid x \in C\left(x_{0}, \alpha\right)\right\}$ is strong equicontinuous and weak uniformly $\mathrm{ACG}^{*}$ on $\mathrm{I}_{\alpha}$. Since $\tilde{x}(\mathrm{t})$ is a generalized solution of the initial value problem (3.9), we have $\tilde{x}(t)$ is continuous. In fact, for all $\lambda \in[0,1], x_{\lambda}^{-}$and $x_{\lambda}^{+}$are continuous. Therefore, for $t_{0} \in[0, a]$ and for all $\varepsilon>0$, there exists $\delta>0$, we have

$$
\left|x_{0}^{-}(\mathrm{t})-x_{0}^{-}\left(\mathrm{t}_{0}\right)\right|<\varepsilon, \quad\left|x_{0}^{+}(\mathrm{t})-x_{0}^{+}\left(\mathrm{t}_{0}\right)\right|<\varepsilon .
$$

For above $t$, there exists a fuzzy number $\tilde{A}$ such that $\tilde{\chi}(t)=\tilde{\chi}\left(t_{0}\right)+\tilde{A}$. Then, we have $\left|A_{0}^{-}\right|<\varepsilon$ and $\left|A_{0}^{+}\right|<\varepsilon$. We notice that

$$
\begin{aligned}
\mathrm{D}\left(\tilde{\mathrm{x}}(\mathrm{t}), \tilde{\mathrm{x}}\left(\mathrm{t}_{0}\right)\right) & =\sup _{\lambda \in[0,1]} \max \left\{\left|x_{\lambda}^{-}(\mathrm{t})-x_{\lambda}^{-}\left(\mathrm{t}_{0}\right)\right|,\left|x_{\lambda}^{+}(\mathrm{t})-x_{\lambda}^{+}\left(\mathrm{t}_{0}\right)\right|\right\} \\
& =\sup _{\lambda \in[0,1]} \max \left\{\left|A_{\lambda}^{-}\right|,\left|A_{\lambda}^{+}\right|\right\}=\max \left\{\left|A_{0}^{-}\right|,\left|A_{0}^{+}\right|\right\}<\varepsilon .
\end{aligned}
$$

So, $\tilde{x}(t)$ is continuous on $[0, a]$. Then, by Theorem 3.14, the initial value problem (3.9) has at least one weak solution $\tilde{x}(t)$.

\section{Conclusions and future works}

In this paper, we study the Henstock-Pettis integral of compact convex set-valued functions and fuzzynumber-valued function and the convergence theorem of fuzzy Henstock-Pettis integrals. In addition, we deal with the Cauchy problem of discontinuous fuzzy systems involving the weak fuzzy Henstock integral in fuzzy number space. The function governing the equations is supposed to be discontinuous with respect to some variables and satisfy nonabsolute fuzzy integrability. Our result improves the result given in $[3,4,6,7,20,22,23,39]$ and [9] (where uniform continuity was required), as well as those referred therein. In the future research, we shall deals with a new derivative and Hestock-Pettis- $\Delta$-integral for fuzzy-number-valued functions on time scales. Also, we shall study and investigate fuzzy differential equations and fuzzy integral equations with $\Delta_{\mathrm{H}}$-derivative and FHP- $\Delta$-integral on time scales. 


\section{Acknowledgment}

The authors would like to thank the editor in chief, the anonymous reviewers and Prof. Xinzhi Liu for providing very helpful comments and suggestions which improved the paper. We also thank to the National Nature Science Foundation of China (Grant No. 11461062, No.11671001, No. 61763044 and No. 61472056), the Ph.D. Research Startup Foundation of Chongqing University of Posts and Telecommunications (No. A2014-90 and No. A2016-13) and Chongqing Municipal Science and technology project (cstc2015jcyjA00015).

\section{References}

[1] J. Banaś, K. Goebel, Measures of noncompactness in Banach spaces, Lecture Notes in Pure and Applied Mathematics, Marcel Dekker, Inc., New York, (1980). 3, 3.13

[2] L. C. Barrosa, L. T. Gomesa, P. A. Tonelli, Fuzzy differential equations: an approach via fuzzification of the derivative operator, Fuzzy Sets and Systems, 230 (2013), 39-52. 1

[3] B. Bede, S. G. Gal, Generalizations of the differentiability of fuzzy-number-valued functions with applications to fuzzy differential equations, Fuzzy Sets and Systems, 151 (2005), 581-599. 1, 2.7, 4

[4] B. Bede, L. Stefanini, Generalized differentiability of fuzzy-valued functions, Fuzzy Sets and Systems, 230 (2013), 119-141. 1, 4

[5] A. M. Bica, One-sided fuzzy numbers and applications to integral equations from epidemiology, Fuzzy Sets and Systems, 219 (2013), 27-48.

[6] Y. Chalco-Cano, H. Román-Flores, On new solutions of fuzzy differential equations, Chaos Solitons Fractals, 38 (2008), 112-119. 4

[7] M.-H. Chen, C.-S. Han, Some topological properties of solutions to fuzzy differential systems, Inform. Sci., 197 (2012), 207-214. 4

[8] M.-H. Chen, D.-H. Li, X.-P. Xue, Periodic problems of first order uncertain dynamical systems, Fuzzy Sets and Systems, $162(2011), 67-78.1$

[9] T. S. Chew, F. Flordeliza, On $x^{\prime}=\mathrm{f}(\mathrm{t}, \mathrm{x})$ and Henstock-Kurzweil integrals, Differential Integral Equations, 4 (1991), 861-868. 1, 4

[10] M. Cichon, Convergence theorems for the Henstock-Kurzweil-Pettis integral, Acta Math. Hungar., 92 (2001), 75-82. 3

[11] M. Cichoń, I. Kubiaczyk, A. Sikorska, The Henstock-Kurzweil-Pettis integrals and existence theorems for the Cauchy problem, Czechoslovak Math. J., 54 (2004), 279-289. 3

[12] P. Diamond, P. Kloeden, Metric spaces of fuzzy sets, Theory and applications, World Scientific Publishing Co., Inc., River Edge, NJ, (1994). 2.4, 2.5, 2.8

[13] L. Di Piazza, K. Musiał, Set-valued Kurzweil-Henstock-Pettis integral, Set-Valued Anal., 13 (2005), 167-169. 1

[14] K. El Amri, C. Hess, On the Pettis integral of closed valued multifunctions, Set-Valued Anal., 8 (2000), 329-360. 1

[15] Z.-T. Gong, On the problem of characterizing derivatives for the fuzzy-valued functions, II, Almost everywhere differentiability and strong Henstock integral, Fuzzy Sets and Systems, 145 (2004), 381-393. 1

[16] Z.-T. Gong, Y.-B. Shao, Global existence and uniqueness of solutions for fuzzy differential equations under dissipative-type conditions, Comput. Math. Appl., 56 (2008), 2716-2723. 1

[17] Z.-T. Gong, Y.-B. Shao, The controlled convergence theorems for the strong Henstock integrals of fuzzy-number-valued functions, Fuzzy Sets and Systems, 160 (2009), 1528-1546. 1

[18] Z.-T. Gong, L.-L. Wang, The Henstock-Stieltjes integral for fuzzy-number-valued functions, Inform. Sci., 188 (2012), 276-297. 1

[19] Z.-T. Gong, C.-X. Wu, Bounded variation, absolute continuity and absolute integrability for fuzzy-number-valued functions, Fuzzy Sets and Systems, 129 (2002), 83-94. 1

[20] M.-S. Guo, X.-Y. Peng, Y.-Q. Xu, Oscillation property for fuzzy delay differential equations, Fuzzy Sets and Systems, 200 (2012), 25-35. 4

[21] R. Henstock, Theory of integration, Butterworths, London, (1963). 1

[22] O. Kaleva, Fuzzy differential equations, Fuzzy Sets and Systems, 24 (1987), 301-317. 1, 2, 4

[23] P. E. Kloeden, T. Lorenz, Fuzzy differential equations without fuzzy convexity, Fuzzy Sets and Systems, 230 (2013), 65-81. 4

[24] I. Kubiaczyk, On a fixed point theorem for weakly sequentially continuous mappings, Discuss. Math. Differential Incl., 15 (1995), 15-20. 3.12

[25] J. Kurzweil, Generalized ordinary differential equations and continuous dependence on a parameter, (Russian) Czechoslovak Math. J., 7 (1957), 418-446. 1, 3, 3

[26] P. Y. Lee, Lanzhou lectures on Henstock integration, Series in Real Analysis, World Scientific Publishing Co., Inc., Teaneck, NJ, (1989). 1, 2.1, 2.2, 3, 3, 3

[27] M. L. Puri, D. A. Ralescu, Fuzzy random variables, J. Math. Anal. Appl., 114 (1986), 409-422. 1 
[28] Š. Schwabik, Generalized ordinary differential equations, Series in Real Analysis, World Scientific Publishing Co., Inc., River Edge, NJ, (1992). 3

[29] S. Schwabik, G.-J. Ye, Topics in Banach space integration, Series in Real Analysis, World Scientific Publishing Co. Pte. Ltd., Hackensack, NJ, (2005). 1, 3

[30] S. Seikkala, On the fuzzy initial value problem, Fuzzy Sets and Systems, 24 (1987), 319-330. 1

[31] Y.-B. Shao, Z.-T. Gong, Discontinuous fuzzy systems and Henstock integrals of fuzzy number valued functions, Int. Conf. Intell. Comput., Springer, Berlin, Heidelberg, 7389 (2012), 65-72. 1

[32] Y.-B. Shao, G.-L. Xue, Discontinuous fuzzy Fredholm integral equations and strong fuzzy Henstock integrals, Articial Intelligence Res., 2 (2013), 87-95.

[33] Y.-B. Shao, H.-H. Zhang, The strong fuzzy Henstock integrals and discontinuous fuzzy differential equations, J. Appl. Math., 2013 (2013), 8 pages.

[34] Y.-B. Shao, H.-H. Zhang, Existence of the solution for discontinuous fuzzy integro-differential equations and strong fuzzy Henstock integrals, Nonlinear Dyn. Syst. Theory, 14 (2014), 148-161.

[35] Y.-B. Shao, H.-H. Zhang, Fuzzy integral equations and strong fuzzy Henstock integrals, Abstr. Appl. Anal., 2014 (2014), 8 pages. 1

[36] C.-X. Wu, Z.-T. Gong, On Henstock integrals of interval-valued functions and fuzzy-valued functions, Fuzzy Sets and Systems, 115 (2000), 377-391. 1, 2.9, 2.12, 2

[37] C.-X. Wu, Z.-T. Gong, On Henstock integral of fuzzy-number-valued functions, I, Fuzzy Sets and Systems, 120 (2001), 523-552. 1, 2.9, 2

[38] C.-X. Wu, M. Ma, Embedding problem of fuzzy number space, II, Fuzzy Sets and Systems, 45 (1992), 189-202. 2.5, 2.6, 2.8

[39] X.-P. Xue, Y.-Q. Fu, Carathéodory solutions of fuzzy differential equations, Fuzzy Sets and Systems, 125 (2002), 239 243. 1,4

[40] B.-K. Zhang, C.-X. Wu, On the representation of n-dimensional fuzzy numbers and their informational context, Fuzzy Sets and Systems, 128 (2002), 227-235. 1 